\title{
Inherited lung cancer: a review
}

\author{
Viviane Teixeira Loiola de Alencar, Maria Nirvana Formiga and Vladmir Cláudio Cordeiro de Lima
}

AC Camargo Cancer Center, R Prof Antônio Prudente, 211 São Paulo, SP 01509-010, Brazil

\begin{abstract}
Lung cancer is the most common cancer worldwide and has high rates of mortality. The major risk factor associated with this disease is tobacco smoke, but approximately $10 \%-$ $25 \%$ of all lung cancer cases occur in patients who have never smoked. Data suggest that lung cancer in never-smokers has a different molecular profile, tumour microenvironment and epidemiology than that in smokers. Several risk factors have been associated with its occurrence, and the possibility of inherited predisposition is becoming clearer. A better understanding of this disease is essential for the future development of personalised screening, diagnosis and treatment approaches, with consequent reduction of mortality. In this review, we discuss historical studies of lung cancer in never-smokers and the currently available evidence of inherited predisposition to this disease.
\end{abstract}

Keywords: hereditary cancer, lung cancer, germline mutation

\section{Introduction}

Lung cancer is the most commonly diagnosed cancer (accounting for $11.6 \%$ of all cases) and the leading cause of cancer death (accounting for $18.4 \%$ of all such deaths) in both sexes worldwide; it is responsible for more than 1.8 million deaths each year [1]. The high mortality rate associated with this disease is attributable in part to its diagnosis at advanced stages, with regional or distant spread, in approximately $80 \%$ of cases; at such stages, treatment is less effective and survival rates are considerably lower (5-year survival, $57.4 \%$ in early stage versus $5.2 \%$ with distant metastasis at diagnosis) [2].

Incidence rates of lung cancer vary among regions as a reflection of different historic patterns of tobacco exposure, which includes exposure intensity and duration, cigarette type and degree of inhalation and the evolution of these patterns over time, commonly called the 'tobacco epidemic'. Recent statistics [1] show that incidence rates among men are the highest in Micronesia/Polynesia, Eastern Asia and much of Europe, especially Eastern Europe. In Africa, the rates remain generally low, although they are intermediate to high in several countries in the northern and southern regions of the continent, notably in Morocco (31.9/100,000) and South Africa (28.2/100,000). Among women, incidence rates are highest in North America, Northern and Western Europe and Australia/New Zealand. Interestingly, the rate among Chinese women is similar to those among women in several Western European countries, such as France, despite substantial differences
Correspondence to: Viviane Alencar Email: vivianetlalencar@gmail.com

ecancer 2020, 14:1008

https://doi.org/10.3332/ecancer.2020.1008

Published: 29/01/2020

Received: $15 / 10 / 2019$

Publication costs for this article were supported by ecancer (UK Charity number 1176307).

Copyright: (c) the authors; licensee ecancermedicalscience. This is an Open Access article distributed under the terms of the Creative Commons Attribution License (http:// creativecommons.org/licenses/by/3.0), which permits unrestricted use, distribution, and reproduction in any medium, provided the original work is properly cited. 
in smoking prevalence between these populations. This rate may be explained by continued exposure to smoke from charcoal burned for heating and cooking purposes [1].

The two major histological forms of lung cancer are non-small cell lung cancer (NSCLC), which accounts for about $85 \%$ of cases, and small cell lung cancer (SCLC). NSCLC can be categorised into three subtypes: squamous cell carcinoma, adenocarcinoma and large-cell carcinoma [3]. Several environmental risk factors are associated with its development, including smoking, passive smoking, air pollution, pulmonary diseases (i.e., tuberculosis, chronic bronchitis), occupational exposure to carcinogenic chemicals, ionising radiation (i.e., radon decay products and $\mathrm{x}$-rays), asbestos and alcohol consumption [4,5]. Tobacco smoking, responsible for more than $80 \%$ of all lung cancer cases in Western populations, increases the relative risk (RR) of lung cancer 10-20-fold in smokers compared with never-smokers [1, 6], with its incidence correlated in a dose-response manner with the cumulative amount and duration of tobacco smoked [7]. Smoking is linked most strongly to SCLC and squamous cell carcinoma, whereas adenocarcinoma is the most common type of lung cancer in patients who have never smoked [3].

According to global statistics, however, only about $10 \%-15 \%$ of all smokers develop lung cancer [8], and 10\%-25\% of all lung cancers are not attributable to smoking $[9,10]$. The latter percentage is even higher, up to $30 \%-40 \%$, in Asian countries [10]. If considered separately, lung cancer in never-smokers (those consuming $<100$ cigarettes/lifetime) would rank as the seventh most common cause of cancer death worldwide, before cancers of the cervix, pancreas and prostate [11]. Environmental tobacco smoke exposure (passive smoking) is a known risk factor in some of these cases, as it has been demonstrated to increase the risk of lung cancer development in non-smokers by at least $20 \%$. Passive smoking, however, is associated with only approximately $16 \%-24 \%$ of lung cancer cases in non-smokers [12]. Today, we know that lung cancers in smokers and never-smokers are two distinct entities, which differ not only epidemiologically but also in their molecular alteration profiles and tumoral microenvironment compositions.

Lung cancer in never-smokers is diagnosed most commonly in women. Patients tend to be younger, respond better to treatment and have better prognoses, due to the more frequent occurrence of actionable mutations that enable specific treatments with receptor tyrosine kinase inhibitors. For example, ALK rearrangements are more common in non-smokers with adenocarcinoma than in smokers with adenocarcinoma or squamous cell carcinoma [13]. Metabolic syndrome also has been linked to the development of lung adenocarcinoma, especially in never-smokers, as genome-wide association studies (GWASs) have demonstrated that EGFR, VTL1A, TNFRSF10C, C3ORF21 and hypermethylations of TNFSF10C, BHLHB5 and BOLL are involved in both pathways [13, 14].

Tumoral microenvironments also appear to be distinct in smokers and never-smokers, as tobacco smoking causes DNA damage in lung epithelial cells and alters the local immune system [13]. In 2018, Li and colleagues [15] performed a study based on 11 lung cancer microarray datasets with samples from 1,111 lung adenocarcinomas and 200 adjacent normal tissues. They found that the pathways involved in carcinogenesis differ in smokers and never-smokers due to differences in outcomes related to mast cells and CD4+ memory T cells, and their active or resting status. Resting mast cells were associated with better prognoses, and their number was found to be decreased in tumour samples relative to those from adjacent normal tissue. On the other hand, activated mast cells (macrophages) and activated CD4+ memory T cells were associated with poor prognoses and were found in greater numbers in tumour samples. The authors observed more resting mast cells and CD4+ memory T cells, both associated with better prognoses, in tissue samples from never-smokers, and more activated cells of these types, correlated with worse prognoses, in samples from smokers [15].

With the current application of policies to control tobacco use worldwide, the proportion of lung cancer cases in never-smokers is expected to increase. Thus, a better understanding of the disease's causal factors, which include hereditary predisposition, is needed.

The essential role of the early detection of lung cancer in the survival rate is well established [2]. Since the publication in 2011 of The National Lung Screening Trial Research [16], which showed a 20\% reduction in mortality from lung cancer with the use of low-dose computed tomography (CT) for the screening of individuals at high risk of the disease, the screening of current and former smokers aged 55-74 years with at least 30 pack-year smoking histories has been recommended [17]. The development of multiple primary malignancies associated with lung cancer has been linked to a hereditary factor and should also be included in more specific patient follow-up protocols [18].

Squamous cell carcinoma, which is usually more prevalent among smokers, also may have a genetic trigger in non-smokers. In 2017, to better understand tumour growth, Park and colleagues [19] analysed the genetic profile of squamous cell carcinoma in non-smokers using comparative genomic hybridisation arrays. They found that the proto-oncogene GAB2 (11q14.1) was frequently amplified in these patients and was likely to contribute to disease development [19]. 
With a better understanding of which non-smokers are at greater risk for lung cancer (e.g., those with inherited susceptibility alleles), the development and application of broader screening guidelines could impact the survival of these individuals. Better elucidation of the physiopathology of these cancers may lead to the development of additional preventive strategies and more accurate approaches to treatment, possibly with new therapeutic targets.

\section{Familial aggregation in lung cancer}

Familial clustering of cancer is characterised by the occurrence of the same type of cancer in two or more first-degree relatives, which may be attributable to inheritable gene mutations. The clinical expression of the disorder is influenced by hereditary predisposition, variable gene penetrance and environmental factors [4]. We estimate today that $5 \%-10 \%$ of all cancers are caused by inherited germline mutations, many of which are associated with known hereditary cancer syndromes [20].

Familial aggregation is the clustering of a disease within families, which may be attributable to genetics, environmental causes or exposure to infectious agents. It is a necessary, although not sufficient, condition for the inference of genetic susceptibility, and studies assessing it are usually first steps in the identification of hereditary diseases. The inherent idea is to determine whether an individual's risk of a disease is enhanced by the existence of relatives with the same illness. It may be inferred by comparing the incidence of a given disease among relatives of affected subjects and in a suitable control group, typically the relatives of healthy subjects [21, 22]. Familial clustering of cancer may be explained by shared environmental factors, inherited mutation of moderate- and high-penetrance genes and/or inherited single nucleotide polymorphisms (SNPs). GWASs, for example, are effective for the detection of common alleles that contribute to the inherited components of common cancers. To assess the genetic effect, many researchers calculate the ratio of the observed incidence of cancer among first-degree relatives to the expected frequency, called lambda $(\lambda)$.

The literature contains several important studies of familial aggregation that has raised the inferred possibility of genetic susceptibility to lung cancer [5, 22]. The first studies to suggest a genetic component for lung cancer were the Baltimore and Buffalo studies performed by Tokuhata and Lilienfeld $[23,24]$. They found a 2.5-fold greater risk of mortality among smoking first-degree relatives of lung cancer probands than among smoking relatives of controls. The Baltimore study [18] also showed that familial aggregation for lung cancer existed with or without the influence of smoking, although the risk of lung cancer development was higher among smokers.

Two studies of familial clustering that have provided important information about specific cancers were based on the Utah family database, from the Utah cancer registry study, which included approximately 125,000 individuals between 1952 and 1992 [25], and the Swedish cancer registry, which included 1,283,047 cancer cases between 1958 and 1997 [26]. In the Utah study, the familial risk of lung cancer was significantly high $[\lambda=2.55,95 \%$ confidence interval (CI) 2.08-3.08]. The study identified significant familial clustering among tobacco-related cancers, such as cancers of the lung, larynx, lip and cervix, which was attributable to shared environmental factors potentially associated with genetic susceptibility. The authors also reported a greater RR for female relatives of female probands than for male relatives of male probands $[11,19]$. The Swedish study showed that the risk of lung cancer in first-degree relatives of 88,589 lung cancer cases was increased by 1.9 times $(95 \% \mathrm{Cl} 1.6-2.4)$, similar to that of other common cancers with well-known genetic components, including breast $(\lambda=1.5)$ and colon $(\lambda=1.9)$ cancers. This type of study does not enable determination of whether the clustering is due to genetic or environmental factors, but the environmental influence was minimised in the Swedish cohort, which included individuals from different generations and thus with different exposures [26].

Another study was performed using data from the Icelandic Cancer Registry on all 2,756 patients in the Icelandic population diagnosed with lung carcinoma between 1955 and 2002 [27]. The familial risk was greater among parents $(\lambda=2.69,95 \% \mathrm{Cl} 2.20-3.23)$, siblings $(\lambda=2.02$, 95\% Cl 1.77-2.23) and children $(\lambda=1.96,95 \% \mathrm{Cl} 1.53-2.39)$, and lesser among second-degree $(\lambda=1.4)$ and third-degree $(\lambda=1.2)$ relatives, although it was significantly higher than expected in the latter groups. For all groups of relatives analysed, the risk of lung cancer was greater for relatives of patients with early-onset disease [27].

More recently, Cannon-Albright et al [28] published the results of another analysis based on the Utah population database, performed to estimate individuals' RRs of lung cancer based on their family histories of the disease. In contrast to the Iceland study, which involved the estimation of risks based on population rates of lung cancer, the researchers used rates of individuals with no family history of this 
malignancy. They identified a total of 5,048 lung cancer cases and estimated that individuals with any family history of lung cancer were more than twice as likely to be diagnosed with lung cancer as were those with no family history. RRs were significant for first-, second- and thirddegree relatives. For first-degree relatives, they ranged from 2.57 (for one or more such relatives) to 4.24 (for three or more such relatives). For second-degree relatives, in the absence of first-degree relatives, RRs ranged from 1.41 (for one or more such relatives) to 4.76 (for four or more such relatives). For third-degree relatives, in the absence of affected first- and second-degree relatives, RRs ranged from 1.18 (for one or more such relatives) to 1.55 (for four or more such relatives). The authors also found a greater risk associated with a first-degree relative's diagnosis at an earlier age (50-60 years). A limitation of this study is that data on tobacco use were not available.

In 2018, Ding and colleagues [29] performed an observational study of the clinicopathological characteristics of 152 patients with the familial lung cancer on the Yunnan-Guizhou Plateau in China. Unique characteristics of these subjects were earlier disease onset; greater frequency of cancer among women, with the most frequent histology of adenocarcinoma; and the tendency to have other cancer histories and present with distant metastasis, consistent with poor prognoses. These findings are controversial when considered together with those of the study on tumour microenvironments and prognosis mentioned above [15].

To better validate whether the familial pattern of a disease is consistent with a genetic model of inheritance due to the existence of a major predisposition gene, a different type of study, such as a segregation analysis, is needed [12]. Two studies [30, 31] have investigated the probability of inheritance of a major gene for lung cancer predisposition, and both showed that the pattern of lung cancer occurrence in families is consistent with Mendelian co-dominant inheritance of a rare autosomal gene. Sellers et al [30] estimated that segregation at this presumed

lung cancer predisposition locus could be responsible for $69 \%$ of lung cancer cases at the age of 50 years, $47 \%$ at the age of 60 years and $22 \%$ at the age of 70 years, possibly as a reflection of an increasing proportion of tobacco exposure-related lung cancer in non-carriers [17, 30]. These studies were important to raise the possibility of a genetic predisposition to lung cancer and to point to the need for further analysis to identify a candidate gene.

\section{Inheritable predisposition genes associated with lung cancer}

\section{Candidate genes associated with lung cancer}

In recent decades, many studies have been performed to gather evidence of a genetic predisposition to lung cancer. Findings from more than 1,000 candidate gene studies have been published in the past 25 years, as such studies are cost effective and relatively easy to perform.

A meta-analysis of 1,018 publications describing 2,910 variants in 754 genes and loci was conducted to interpret genetic associations of common variants with lung cancer [32]. Credibility assessment of the accumulated epidemiological evidence from 246 eligible variants suggested that eight genetic variants (APEX1 rs1760944, AXIN2 rs2240308, CHRNA3 rs6495309, CXCR2 rs1126579, CYP2E1 rs6413432, HYKK rs931794, PON1 rs662 and REV3L rs462779) were strongly associated with the risk of lung cancer development, and ten (ATM rs189037, CD3EAP rs967591, CYP2A6 rs1801272, HIF1A rs11549467, PDCD5 rs1862214, PROM1 rs2240688, TP53 rs12951053, TP63 rs10937405, WWOX CNV-67048 and XRCC1 rs3213255) were moderately associated with this risk. An analysis based on the histological type of cancer revealed significant associations for 25 variants in the NSCLC group, eight of which (CHRNA5 rs16969968, CLPTM1L rs402710, CYP2E1 rs6413432, ERCC1 rs11615, FGFR4 rs351855, HYKK rs931794, MIR146A rs2910164 and TERT rs2736098) demonstrated strong cumulative epidemiological evidence. Five variants in the SCLC group showed significant associations, but these associations were moderate (CHRNA5 rs16969968, CYP1A1 rs4646903 and NQO1 (rs1800566) and weak (GSTM1 present/null and XPC rs2228001) [32], which may point to a greater contribution of genetic predisposition in patients with NSCLC than in those with SCLC.

A candidate gene study published in 2017 employed germline sequence data from 555 lung adenocarcinoma cases from The Cancer Genome Atlas to evaluate possible risks associated with the ATM serine/threonine kinase gene (ATM), the BRCA2 DNA repair-associated gene (BRCA2), the checkpoint kinase 2 gene (CHEK2), EGFR, the parkin RBR E3 ubiquitin protein ligase gene (PARK2), the telomerase reverse transcriptase gene (TERT), the tumour protein p53 gene (TP53) and the YES-associated protein 1 gene (YAP1). They found 14 pathogenic mutations in five genes with a frequency of $2.5 \%$. These mutations occurred more frequently in genes associated with the DNA repair pathway, such as ATM (50\%), followed by TP53, BRCA2, EGFR and PARK2 [33]. 


\section{GWASs}

More recently, GWASs have been developed as alternatives to candidate gene studies, as they provide broader coverage of genetic variation through the genotyping of up to 1,000,000 genetic variants or SNPs [5]. They are highly powered to enable the identification of common risk alleles, which are usually of low penetrance [12]. Three GWASs for lung cancer [from the International Agency for Research on Cancer (IARC), MD Anderson Cancer Center, and deCODE Genetics], published in 2008, identified the same locus in chromosome region 15q25, an area that includes a cluster of nicotinic acetylcholine receptor genes (CHRNA3, CHRNA5 and CHRNB4) and is associated strongly with lung cancer [34-36]. The IARC study was later expanded, leading to the discovery of an additional susceptibility locus on chromosome 5p, in a region that includes TERT and the cleft lip and palate transmembrane 1-like gene (CLPTMIL) [37]. The IARC and MD Anderson studies linked these genetic regions directly to lung cancer risk, and the deCODE study identified a sequence variant in the cluster of nAChR genes on chromosome 15 that was associated with smoking quantity, and thus indirectly to elevated cancer risk [34-36]. In a GWAS reported in 2019, Hung et al [38] detected three SNPs (rs31490, rs380286 and rs4975616), also mapped to the 5p15.33 CLPTM1-like TERT region, which were linked to genetic susceptibility to lung cancer in a European cohort of never-smokers.

The association between $15 q 25$ and lung cancer seems to be strong with an $80 \%$ increase in the risk of lung cancer development for individuals who inherit two alleles [5], which is probably not due solely to the influence of tobacco. In 2009, findings of an analysis of Chinese two-stage case-control studies (3,500 lung cancer cases and 3,300 controls) were published [39]. The three alleles identified in the studies of Caucasian populations mentioned above [34-36] were found to be rare in the Asian population, but the study demonstrated an increased risk of lung cancer associated with four other SNPs in 15q25 ( $r$ 2036534C > T, rs667282C > T, rs12910984G > A and rs6495309T > C). This effect was similar among never-smokers and ever-smokers and was independent of sex [39].

In 2014, a GWAS of data from more than 20,000 cases and 40,000 controls revealed a strong association of the risk of squamous cell carcinoma development with rare variants of BRCA2-K3326X [rs11571833; odds ratio (OR) $=2.47, p=4.74 \times 10^{-20}$ and CHEK2-I157 ( $r$ 17879961; OR $=0.38, p=1.27 \times 10^{-13}$ ) [40]. For adenocarcinoma histology, the authors described a common variation at 3q28 (TP63; rs13314271; OR $=1.13, p=7.22 \times 10^{-10}$ ) previously associated with cancer only among Asians.

A study published in 2018 showed that chromosome 15q25 modifies lung cancer risk and is involved in the pathogenesis of this disease via activation of the neuroactive ligand receptor interaction pathway. This pathway, which is also associated with neuropsychiatric disorders, congenital diseases and nicotine dependence, consists mainly of a group of neuroreceptor genes and is involved in environmental information processing and signalling molecules and interactions [41].

Other variants associated with lung cancer susceptibility in GWASs are 6p21 and $5 \mathrm{p} 15$ [42, 43]. A meta-analysis of 16 GWASs including approximately 14,900 lung cancer cases and 29,485 controls of European descent identified risk loci at 5p15, 6p21 and 15q25 with histology-specific effects for the first two variants [44]. The researchers also identified a locus for squamous cell carcinoma susceptibility at 9p21 [44].

\section{Linkage analysis studies}

As GWASs tend to identify common risk alleles of low penetrance, based on the common disease-common variant hypothesis (i.e., that a small number of common risk alleles confer small to moderate risks of disease), they have explained only a small portion of the hereditary risk of lung cancer [45]. With the aim of identifying high-penetrance genes with stronger effects, linkage analyses have also been performed. These analyses are based on the rationale that some genetic traits are attributable to genes that are linked, and thus inherited together. This type of study helps to identify whether a disease phenotype is caused by the mutation in one gene alone, or whether mutations in other genes may be responsible for a similar or identical phenotype [46]. In 2004, Bailey-Wilson et al [47] published a genome-wide linkage study, performed using an autosomal-dominant model, of 52 families of probands with lung cancer who had several first-degree relatives with the same disease; they identified a major susceptibility locus related to lung cancer risk in the 6q23-25 chromosomic region. This study proved that significant gene linkage for familial lung cancer exists, suggesting that some rare genetic variants play important roles in lung cancer susceptibility. 


\section{Sanger sequencing and whole-genome sequencing}

Based on these findings, another hypothesis becomes appealing: the common disease-rare variant hypothesis, according to which risk loci contain multiple rare independent risk alleles across populations, each with moderate to high penetrance (high allelic heterogeneity). To explore this hypothesis, direct sequencing is the better option, as it enables the identification of all rare alleles in a population. Sanger sequencing, also known as 'first-generation sequencing', was the first technique used to sequence the human genome. It is still used widely for the sequencing of individual genes, but, due to its high cost, 'second-generation sequencing' or next-generation sequencing (NGS) with multi-gene panels is now performed [45]. NGS has been adopted widely and can be used to sequence more target genes with less DNA and reduced cost, time and labour than required for Sanger sequencing.

In the following paragraphs, we explore the main genes that are possibly related to hereditary lung cancer, discovered in studies based on these two techniques.

\section{Genes associated with inherited lung cancer syndromes}

\section{EGFR T790M}

Case reports have described germline mutations in the kinase domain of the EGFR, such as R776G, R776H, T790M, V843I and P848L, which confer a greater risk of cancer development. Among these, T790M seems to be associated with a specific lung cancer syndrome targeting never-smokers [48]. In various studies, Sanger sequencing has correlated familial clustering of lung cancer with a germline T790M mutation in EGFR [49-56]. This mutation is rare, but EGFR is considered to be a major cancer predisposition gene, associated with an estimated 31\% risk of lung cancer development in non-smoking carriers [52]. Although the presence of the EGFR T790M mutation seems to be sufficient to induce tumour formation in mouse models [56], this mutation is characterised as weakly oncogenic. When associated with a common activating EGFR mutation, however, the oncogenic potential is significantly enhanced, possibly contributing to earlier disease onset. Seventythree percent of patients with lung cancer and the germline T790M mutation have been found to carry a second activating mutation [51], most commonly the L858R mutation [49].

The germline T790M EGFR mutation is present in approximately 1\% of NSCLC cases [52] with a median age at diagnosis of 40 years, and the most common histology is adenocarcinoma. Although somatic mutations in EGFR are more common among patients from East Asia, the prevalence of germline mutations in lung cancer cases in Japan was lower than that in North America [51], and, to our knowledge, no case of germline T790M EGFR mutation in East Asia has been reported in the literature. Sporadic EGFR mutation and germline T790M EGFR mutation are associated more frequently with female sex and non-smoking status. The most frequent presentation in CT scans is bilateral ground-glass opacities and pulmonary nodules with an indolent disease course [51]. The screening of unselected populations has no proven benefit, but, as the germline T790M EGFR mutation is present in approximately 50\% of patients with baseline T790M EGFR identified in pre-treatment evaluation [52], some authors have suggested that these patients should be referred for routine germline genotyping to identify carriers [42, 44].

Recently, the Dana-Farber Institute performed the INHERIT EGFR Study, which investigated families with this variant. Among the 105 participants, germline EGFR mutations were found in 63\% of patients with EGFR T790M detected in lung cancer tissue at diagnosis, and in 62\% (16 of 27) and $44 \%$ ( 4 of 9) of first- and second-degree relatives of germline carriers, respectively. The affected individuals had a median age at lung cancer diagnosis of 57 (range 28-82) years; $81 \%$ were white and $19 \%$ were black; $52 \%$ were never-smokers; $65 \%$ of cases were diagnosed at advanced stage (IV); and imaging analysis suggested an indolent multifocal nodular phase, progressing to lymph-node and then remote metastatic disease. Tumour genotyping revealed somatic EGFR co-mutations in 94\% of probands: 6 exon 19 del, 12 L858R, 6 G719X, 1 exon 19 del and G719R, 1 L861Q, 2 H773R and 1 V774M. Patients who were treated with osimertinib presented no unexpected toxicity [57].

\section{TP53 (Li-Fraumeni syndrome)}

Li-Fraumeni Syndrome (LFS) is a rare hereditary condition associated with a germline mutation of TP53 that predisposes the patient to the occurrence of cancers in multiple organs, usually with early onset. Statistics show that approximately $50 \%$ of carriers will develop cancer by the age of 30 years with lifetime risks of up to $70 \%$ in men and $100 \%$ in women [58]. 
Tumours classically associated with this syndrome include breast cancer, soft-tissue sarcoma, osteosarcoma, brain tumours and adrenocortical carcinoma. Lung cancer occurs in $2.3 \%-6.8 \%$ of patients with LFS [58], most frequently men, with a median age at diagnosis of 48 years [59]. Lung cancer is not included in the classic LFS criteria (proband diagnosed with sarcoma at $<45$ years of age, first-degree relative with cancer diagnosed at age $<45$ years, and first- or second-degree relative with cancer onset at age $<45$ years or sarcoma at any age). In 2009, an extended version of the Chompret criteria was published, which included lung cancer in the LFS tumour spectrum, as follows: a proband with a single tumour of LFS spectrum (sarcoma, premenopausal breast cancer, brain tumour, adrenocortical, leukaemia or lung [bronchoalveolar] cancer) diagnosed at age $<46$ years and at least one first-degree or second-degree relative with a tumour of LFS spectrum (except breast cancer if the proband has breast cancer) with onset at age $<56$ years or with multiple tumours; OR a proband with multiple tumours (except multiple breast cancers), two of which are tumours of LFS spectrum, with the first occurring at age < 46 years; OR a proband diagnosed with an adrenocortical carcinoma or choroid plexus carcinoma, irrespective of family history [58].

In 2017, preliminary data from the LIFESCREEN randomised clinical trial revealed a greater frequency of lung adenocarcinoma [22\% (five patients, most non-smokers)] among patients with the syndrome [60]. In an analysis of 555 lung adenocarcinoma cases from The Cancer Genome Atlas, 14 pathogenic mutations in five genes were identified. Among patients with TP53 mutations, the most common variants found were TP53 R267Q, TP53 P152L and TP53 R158L, all previously linked to LFS [33].

In some reports, EGFR mutations have been detected in patients with LFS who develop lung cancer; the loss of p53 function may free the promoter of the EGFR gene and make the gene more susceptible to the occurrence of mutations [61].

\section{BRCA}

BRCA mutations have always been associated more frequently with breast and ovarian cancer syndrome, but a 2017 study showed that the frequencies of other cancers were higher among subjects in hereditary than in non-hereditary branches of the families of patients eligible for BRCA testing, especially in the lung, kidney, liver and larynx [62]. Members of hereditary family branches had greater probabilities of lung cancer $(\mathrm{OR}=4.5,95 \% \mathrm{Cl} 2.15-9.38)$, liver cancer $(\mathrm{OR}=4.02$, 95\% $\mathrm{Cl} 1.14-14.15)$ and laryngeal cancer $(\mathrm{OR}=3.4,95 \% \mathrm{Cl} 1.12-10.39)$, independent of sex and age. These findings are important not only to guide the application preventive measures but also for the future development of possible therapeutic approaches.

\section{Human epidermal growth factor 2 (HER2)}

HER2 is an oncogene in the EGFR family. It is overexpressed in patients with breast cancer at a frequency of 15\%-20\% [63]. In lung cancer, however, somatic mutation of HER2 is rare. Such mutations are found in $1.6 \%-2.5 \%$ of NSCLC cases [64]. They are more frequent among nonsmokers, adenocarcinomas, patients of Asian ethnicity and females [65] and are associated with poor treatment response [64].

A Japanese study published in 2013 was the first to describe germline HER2 mutations in the transmembrane domain as conferring potential susceptibility to lung cancer [65]. The germline variant G660D was identified through whole-exome sequencing in a Japanese family with a high rate of lung cancer. The proband was a woman who was a light smoker with a history of multiple lung adenocarcinomas at the age of 44 years who was treated with lobectomy, but subsequently recurred. At the time of the study, she was 53 years old and had multiple bilateral lung adenocarcinomas. With the hypothesis that mutations in the transmembrane domain of HER2 act as drivers, the researchers sequenced exon 17 of HER2 in samples of 315 sporadic NSCLCs, 253 of which were adenocarcinomas, and identified a novel non-synonymous somatic mutation, V659E, in one patient. These data, however, are not definitive for the attribution of carcinogenic potential to these mutations, and further studies are needed [66].

\section{YAP1}

YES-associated protein 1 is a transcriptional coactivator that influences tissue growth and organ size. In 2015, a germline mutation (YAP R331W) in the transactivation domain was identified by NGS of samples from a family with lung adenocarcinoma clustering and validated in an external cohort of 1,135 participants without cancer and 1,312 patients with lung adenocarcinoma [66]. The proband of the original family was a mother of four affected daughters and one unaffected son. In this family, individuals in whom YAP1 mutation was detected (smokers 
and never-smokers, aged 50-89 years) had been diagnosed with lung adenocarcinoma or were being monitored due to the detection of ground-glass opacities. This allele was associated with a 5.9-fold increased risk of lung cancer development [67].

A study published in 2017 demonstrated that YAP acts as an EGFR downstream signalling molecule, modulating lung cell proliferation, and is a potential therapeutic target for EGFR-dependent adenocarcinomas [68].

\section{CHEK2}

Kukita et al [69] reported on two siblings with histories of multiple primary lung cancers at the age of 60 years. The female patient subsequently developed breast cancer, and the male patient had a history of prostate and colon cancers. Exome analysis revealed non-synonymous homozygous mutations in CHEK2, FCGRT, INPP5J, MYO18B and SFI1. The mutation in CHEK2 altered the tertiary structure of CHK2 by disrupting the salt bridge between p.R474 and p.E394, making it unstable. This effect appeared to have a causative relationship with the familial cancer described, as no structural change was observed in the other mutated genes. However, further studies need to be performed to establish the pathogenic role of this CHEK2 mutation in human carcinogenesis.

\section{Other genes}

Kanwal et al [70] analysed germline mutations associated with the familial lung cancer in nine subjects (four patients with lung cancer and five healthy relatives) using whole-genome sequencing. Among the 12 most highly mutated genes, as validated by a polymerase chain reaction and DNA sequencing, they identified five genes (ARHGEF5, ANKRD20A2, ZNF595, ZNF812 and MYO18B) with germline mutations potentially associated with lung cancer development. Some mutations in the MUC12, FOXD4L3 and FOXD4L5 genes occurred at greater frequencies in samples from subjects with the familial lung cancer or in lung cancer tissue compared with samples from healthy subjects. The authors also found germline mutations in many genes of non-coding RNA.

In a study published in 2018, whole-genome sequencing of samples from 36 never-smoking Chinese patients with lung adenocarcinoma diagnosed at $\leq 45$ years of age identified 4,344-60,259 somatic mutations per tumour [70]. To evaluate genetic predisposition risk, germline variants from the 36 initial patients and 28 additional lung adenocarcinoma cases were analysed; the frequency of pathogenic and likely pathogenic germline mutations among young patients was 78.3\%. The main germline mutations identified were BPIFB1 (rs6141383, p.V284M), CHD4 (rs74790047, p.D140E), PARP1 (rs3219145, p.K94OR), NUDT1 (rs4866, p.V83M), RAD52 (rs4987207, p.S346*) and MFI2 (rs17129219, p.A559T). A TP53 missense germline mutation (rs121912664, p. R205H) was found in one individual with a tumour that appeared to be hypermutated relative to the others. As the overall survival of these patients was not altered by the presence of germline mutations, their mutations should not be considered to be predictive of prognosis [71].

\section{Discussion}

Genetic counselling for lung cancer remains a challenge. No guideline for genetic testing has been established based solely on a family history of lung cancer in non-smokers, unless the proband fulfils criteria for a known hereditary syndrome.

In addition, genetic testing options for lung cancer remain very limited. Most commercial laboratories offer limited or no testing for hereditary lung cancer variants, and no guideline has been established for the best way to manage unaffected carriers of variants associated with lung cancer, such as germline EGFR T790M, HER2 and YAP1 mutations. Screening with low-dose CT may be incorporated into the care of these patients, but the optimum frequency of such evaluation is uncertain.

\section{Conclusions}

Lung cancer in never-smokers is the seventh most lethal cancer worldwide, and its incidence will increase in the upcoming years, as the tobacco burden is falling rapidly in many countries. Much remains to be learned about this pathology, which has proven to be very different 
from lung cancer related to smoking, not only epidemiologically but also at the molecular level. Many affected patients show clear hereditary predisposition, and several genes have been associated with an increased risk of lung cancer development. As the prognosis is related closely to the disease stage at diagnosis, a better understanding of the genetic factors associated with lung cancer in never-smokers and of when genetic counselling is indicated is needed to provide effective screening methods and detect asymptomatic carriers in predisposed families. More research is warranted to provide evidence-based screening guidelines for carriers of germline lung cancer risk alleles.

\section{Funding statement}

No funding was received for this article.

\section{Conflicts of interest statement}

The authors declare no conflicts of interest.

\section{References}

1. Bray F, Ferlay J, and Soerjomataram I, et al (2018) Global cancer statistics 2018 : GLOBOCAN estimates of incidence and mortality worldwide for 36 cancers in 185 countries CA Cancer J Clin 68 394-424 https://doi.org/10.3322/caac.21492 PMID: 30207593

2. National Cancer Institute (2019) SEER Cancer Statistics Review, 1975-2016 [https://seer.cancer.gov/csr/1975_2016/]

3. Herbst R, Heymach J, and Lippman SM (2008) Molecular origins of cancer (lung cancer) N Engl J Med 359 1367-1380 https://doi. org/10.1056/NEJMra0802714 PMID: 18815398

4. Kanwal M, Ding X, and Cao Y (2017) Familial risk for lung cancer Oncol Lett 13 535-542 https://doi.org/10.3892/ol.2016.5518 PMID: 28356926 PMCID: 5351216

5. Brennan P, Hainaut P, and Boffetta P (2011) Genetics of lung-cancer susceptibility Lancet Oncol 12 399-408 https://doi.org/10.1016/ S1470-2045(10)70126-1

6. Brownson R, Alavanja M, and Caporaso N, et al (1998) Epidemiology and prevention of lung cancer in nonsmokers Epidemiol Rev 20(2) 218-236 https://doi.org/10.1093/oxfordjournals.epirev.a017982

7. Doll R, Peto R, and Wheatley K, et al (1994) Mortality in relation to smoking : 40 years' observations on male British doctors Br Med J 309(6959) 901-911 https://doi.org/10.1136/bmj.309.6959.901

8. Mattson M, Pollack E, and Cullen JW (1987) What are the odds that smoking will kill you ? Am J Public Health 77(4) 425-431 https:// doi.org/10.2105/AJPH.77.4.425 PMID: 3826460 PMCID: 1646951

9. Scagliotti G, Longo M, and Novello S (2009) Nonsmall cell lung cancer in never smokers Curr Opin Oncol 21 99-104 https://doi. org/10.1097/CCO.0b013e328321049e PMID: 19532009

10. Lee $\mathrm{Y}$, Kim J, and Kim S, et al (2011) Lung cancer lung cancer in never smokers : change of a mindset in the molecular era Lung Cancer 72(1) 9-15 https://doi.org/10.1016/j.lungcan.2010.12.013 PMID: 21272954

11. Sun S, Schiller J, and Gazdar AF (2007) Lung cancer in never smokers - a different disease Nature 7(10) 778-790

12. Musolf A, Simpson C, and Andrade M, et al (2017) Familial lung cancer : a brief history from the earliest work to the most recent studies Genes (Basel) 8(36) 1-13 https://doi.org/10.3390/genes8010036

13. Smolle E and Pichler M (2019) Non-smoking-associated lung cancer : a distinct entity in terms of tumor biology, patient characteristics and impact of hereditary cancer predisposition Cancers (Basel) 11(204) 1-13 https://doi.org/10.3390/cancers11020204 
14. Okazaki I, Ishikawa S, and Sohara Y (2014) Genes associated with succeptibility to lung adenocarcinoma among never smokers suggest the mechanism of disease Anticancer Res 34 5229-5240 PMID: 25275015

15. Xufan L, Wu J, and Zhou L, et al (2018) Smoker and non-smoker lung adenocarcinoma is characterized by distinct tumor immune microenvironments Oncoimmunology 7(10) 1-11 [https://doi.org/10.1080/2162402X.2018.1494677]

16. The National Lung Screening Trial Research Team, Aberle DR, and Adams AM, et al (2011) Reduced lung-cancer mortality with lowdose computed tomographic screening N Engl J Med 365(5) 395-409 https://doi.org/10.1056/NEJMoa1102873 PMID: 21714641 PMCID: 4356534

17. Smith R, Andrews K, and Brooks D, et al (2018) Cancer screening in the United States, 2018 : a review of current American Cancer Society Guidelines and current issues in cancer screening CA Cancer J Clin 68(4) 297-316 https://doi.org/10.3322/caac.21446 PMID: 29846940

18. Haraguchi S, Koizumi K, and Hioki M, et al (2007) Hereditary factors in multiple primary malignancies associated with lung cancer Surg Today 37 375-378 https://doi.org/10.1007/s00595-006-3420-5 PMID: 17468817

19. Park Y, Bae S, and Ji W, et al (2017) GAB2 amplification in squamous cell lung cancer of non-smokers J Korean Med Sci 32 1784-1791 https://doi.org/10.3346/jkms.2017.32.11.1784 PMID: 28960030 PMCID: 5639058

20. Ramirez-Calvo M, Casado Z, and Fernandez-Serra, et al (2019) Implementation of massive sequencing in the genetic diagnosis of hereditary cancer syndromes : diagnostic performance in the hereditary cancer programme of the valencia community (FamCan-NGS) BioMed Cent 17 1-7

21. Matthews A, Finkelstein D, and Betensky AR (2008) Analysis of familial aggregation studies with complex ascertainment schemes Stat Med 27(24) 5076-5092 https://doi.org/10.1002/sim.3327 PMID: 18618413 PMCID: 2562890

22. Risch N (2001) The genetic epidemiology of cancer : interpreting family and twin studies and their implications for molecular genetic approaches Cancer Epidemiol Biomarkers Prev 10 733-741 PMID: 11440958

23. Tokuhata G and Lilienfeld AM (1963) Familial aggregation of lung cancer among hospital patients Public Health Rep 78(4) 277-284 https://doi.org/10.2307/4591778 PMID: 13985328 PMCID: 1915246

24. Tokuhata $G$ and L ilienfeld AM (1963) Familial aggregation of lung cancer in humans J Natl Cancer Inst 30(2) 289-312 PMID: 13985327

25. Goldgar D, Easton D, and Cannon-Albright LA, et al (1994) Systematic population-based assessment of cancer risk in first-degree relatives of cancer probands J Natl Cancer Inst 86(21) 1600-1608 https://doi.org/10.1093/jnci/86.21.1600 PMID: 7932824

26. Lindelof $B$ and Eklund $G$ (2001) Analysis of hereditary component of cancer by use of a familial index by site Lancet 358 1696-1698 https://doi.org/10.1016/S0140-6736(01)06721-6 PMID: 11728548

27. Jonsson S, Thorsteinsdottir U, and Gudbjartsson DF, et al (2004) Familial risk of lung carcinoma in the icelandic population JAMA 292 2977-2983 https://doi.org/10.1001/jama.292.24.2977 PMID: 15613665

28. Cannon-Albright L, Carr SR, and Akerleym W (2019) Population-based relative risks for lung cancer based on complete family history of lung cancer J Thorac Oncol 14(7) 1184-1191 https://doi.org/10.1016/j.jtho.2019.04.019 PMID: 31075544 PMCID: 6592768

29. Ding $X$, Chen $Y$, and Yang J, et al (2018) Characteristics of familial lung cancer in yunnan-guizhou plateau of Front Oncol 8(637) 1-10 https://doi.org/10.3389/fonc.2018.00637

30. Sellers TA, Bailey-Wilson JE, and Elston RC, et al (1990) Division of Epidemiology, School of Public Health, University of Minnesota, Health Sciences Unit A, 515 Delaware St., S.E., Minneapolis M. Evidence of mendelian inheritance in the pathogenesis of lung cancer J Natl Cancer Inst 82 1272-1279 https://doi.org/10.1093/jnci/82.15.1272 PMID: 2374177

31. Yang P, Schwartz AG, and Mcallister AE, et al (1999) Lung cancer risk in families of nonsmoking probands : heterogeneity by age at diagnosis Genet Epidemiol 17 253-273 PMID: 10520209 
32. Wang J, Liu Q, and Yuan S, et al (2017) Genetic predisposition to lung cancer : comprehensive literature integration, meta-analysis, and multiple evidence assessment of candidate-gene association studies Nature 7 1-13

33. Parry EM, Gable DL, and Stanley SE, et al (2017) Germline mutations in DNA repair genes in lung adenocarcinoma J Thorac Oncol 12(11) 1673-1678 https://doi.org/10.1016/j.jtho.2017.08.011 PMID: 28843361 PMCID: 5659909

34. Hung RJ, Mckay JD, and Gaborieau V, et al (2008) A susceptibility locus for lung cancer maps to nicotinic acetylcholine receptor subunit genes on 15q25 Nature 452 633-637 https://doi.org/10.1038/nature06885 PMID: 18385738

35. Amos $\mathrm{Cl}$, Wu X, and Broderick P, et al (2008) Genome-wide association scan of tag SNPs identifies a susceptibility locus for lung cancer at 15q25.1 Nat Genet 40(5) 616-622 https://doi.org/10.1038/ng.109 PMID: 18385676 PMCID: 2713680

36. Thorgeirsson TE, Geller F, and Sulem P, et al (2008) A variant associated with nicotine dependence, lung cancer and peripheral arterial disease Nature 452(7187) 638-642 https://doi.org/10.1038/nature06846 PMID: 18385739 PMCID: 4539558

37. Mckay JD, Hung RJ, and Gaborieau V, et al (2009) Lung cancer susceptibility locus at 5p15.33 Nat Genet 40(12) 1404-1406 https://doi. org/10.1038/ng.254 PMCID: 2748187

38. Hung RJ, Spitz MR, and Houlston RS, et al (2019) Lung cancer risk in never-smokers of European descent is associated with genetic variation in the 5p 15. 33 TERT-CLPTM1LI region J Thorac Oncol 14(8) 1360-1369 https://doi.org/10.1016/j.jtho.2019.04.008 PMID: 31009812 PMCID: 6833942

39. Wu C, Hu Z, and Yu D, et al (2009) Genetic variants on chromosome 15q25 associated with lung cancer risk in chinese populations Cancer Res 69(12) 5065-5073 https://doi.org/10.1158/0008-5472.CAN-09-0081 PMID: 19491260

40. Wang Y, McKay JD, and Rafnar T, et al (2014) Rare variants of large effect in BRCA2 and CHEK2 affect risk of lung cancer Nat Genet 46(7) 736-741 https://doi.org/10.1038/ng.3002 PMID: 24880342 PMCID: 4074058

41. Xuemei J, Yohan B, and Maria TL, et al (2018) Identification of susceptibility pathways for the role of chromosome 15q25.1 in modifying lung cancer risk Nature 9 1-15

42. Wang $Y$, Broderick $P$, and Webb E, et al (2008) Common 5p15.33 and 6p21.33 variants influence lung cancer risk Nat Genet 40(12) 1407-1409 https://doi.org/10.1038/ng.273 PMID: 18978787 PMCID: 2695928

43. Amos Cl, Pinney SM, and Li Y, et al (2010) A susceptibility locus on chromosome 6q greatly increases lung cancer risk among light and never smokers Cancer Res 70(6) 2359-2368 https://doi.org/10.1158/0008-5472.CAN-09-3096 PMID: 20215501 PMCID: 2855643

44. Timofeeva MN, Hung RJ, and Rafnar T, et al (2012) Influence of common genetic variation on lung cancer risk : meta-analysis of 14900 cases and 29485 controls Hum Mol Genet 21(22) 4980-4995 https://doi.org/10.1093/hmg/dds334 PMID: 22899653 PMCID: $\underline{3607485}$

45. Yang IV and Schwartz DA (2012) The next generation of complex lung genetic studies Am J Respir Crit Care Med 186(11) 1087-1094 https://doi.org/10.1164/rccm.201207-1178PP PMID: 22936355 PMCID: 3530203

46. Stefan MP (1999) Genetic linkage analysis Jama Neurol 56 667-672

47. Bailey-Wilson JE, Amos Cl, and Pinney SM, et al (2004) A major lung cancer susceptibility locus maps to chromosome 6q23-25 Am J Hum Genet 75 460-474 https://doi.org/10.1086/423857 PMID: 15272417 PMCID: 1182024

48. Yamamoto H, Yatabe $\mathrm{Y}$, and Toyooka S (2018) Inherited lung cancer syndromes targeting never smokers Transl Lung Cancer Res 20(4) 498-504 https://doi.org/10.21037/tlcr.2018.06.01

49. Lou Y, Pecot CV, and Tran HT, et al (2016) Case report: germline mutation of T790M and dual/multiple EGFR mutations in patients with lung adenocarcinoma Clin Lung Cancer 17(2) 1-13 https://doi.org/10.1016/j.cllc.2015.11.003 
50. Thomas A, Xi L, and Carter CA, et al (2013) Concurrent molecular alterations in tumors with germ line epidermal growth factor receptor T790M mutations Clin Lung Cancer 14(4) 452-456 https://doi.org/10.1016/j.cllc.2013.01.005 PMID: 23540867 PMCID: 5002228

51. Gazda A, Robinson L, and Oliver D, et al (2014) Hereditary lung cancer syndrome targets never smokers with germline egfr gene T790M mutations J Thorac Oncol 9(4) 456-463 https://doi.org/10.1097/JTO.0000000000000130

52. Yu HA, Arcila ME, and Fleischut MH, et al (2014) Germline EGFR T790M mutation found in multiple members of a familial cohort J Thorac Oncol 9(4) 554-558 https://doi.org/10.1097/JTO.0000000000000052 PMID: 24736080 PMCID: 4412273

53. Oxnard GR, Miller VA, and Robson ME, et al (2012) Screening for germline EGFR T790M mutations through lung cancer genotyping J Thorac Oncol 7(6) 1049-1052 https://doi.org/10.1097/JTO.0b013e318250ed9d PMID: 22588155 PMCID: 3354706

54. Lung $C$ and Patients $C$ (2011) Inherited germline T790M mutation and somatic epidermal growth factor receptor mutations in nonsmall cell lung cancer patients J Thorac Oncol 6(2) 395-396 https://doi.org/10.1097/JTO.0b013e3182059a6f

55. Bell DW, Gore I, and Okimoto RA, et al (2005) Inherited susceptibility to lung cancer may be associated with the T790M drug resistance mutation in EGFR Nat Genet 37(12) 1315-1316 https://doi.org/10.1038/ng1671 PMID: 16258541

56. Regales L, Balak MN, and Gong Y, et al (2007) Development of new mouse lung tumor models expressing EGFR T790M mutants associated with clinical resistance to kinase inhibitors PLoS One 2(8) 1-10 https://doi.org/10.1371/journal.pone.0000810

57. Oxnard G, Heng J, and Chen R, et al (2017) Final report of the INHERIT EGFR study - 33 unrelated kindreds carrying germline EGFR mutations J Thorac Oncol 12(11) S1758 https://doi.org/10.1016/j.jtho.2017.09.355

58. McBride KA, Ballinger ML, and Killick E, et al (2014) Li-Fraumeni syndrome: cancer risk assessment and clinical management Nat Rev Clin Oncol 11(5) 260-271 https://doi.org/10.1038/nrclinonc.2014.41 PMID: 24642672

59. Amadou A, Waddington MI, and Hainaut $P(2017)$ Revisiting tumor patterns and penetrance in germline TP53 mutation carriers : temporal phases of Li-Fraumeni syndrome Curr Opin Oncol 29(00) 1-7

60. Ballinger ML, Ferris NJ, and Moodie K, et al (2017) Lung adenocarcinoma as part of the Li-Fraumeni syndrome spectrum : preliminary data of the LIFSCREEN randomized clinical trial overestimating the benefit of cancer drugs JAMA Oncol 3(12) 4-5

61. Michalarea V, Calcasola M, and Cane P, et al (2014) Lung Cancer EGFR-mutated lung cancer in Li-Fraumeni syndrome Lung Cancer 85 485-487 https://doi.org/10.1016/j.lungcan.2014.06.017 PMID: 25047674

62. Digennaro M, Sambiasi D, and Tommasi S, et al (2017) Hereditary and non-hereditary branches of family eligible for BRCA test: cancers in other sites Hered Cancer Clin Pract 15(7) 1-5 https://doi.org/10.1186/s13053-017-0067-8

63. Marano L and Roviello F (2015) The distinctive nature of HER2-positive gastric cancers Eur J Surg Oncol 41(3) 271-273 https://doi. org/10.1016/j.ejso.2014.12.007 PMID: 25605551

64. Koeller DR, Chen R, and Oxnard GR (2018) Hereditary lung cancer risk : recent discoveries and implications for genetic counseling and testing Curr Genet Med Rep 6 83-88 https://doi.org/10.1007/s40142-018-0140-2

65. Shigematsu $\mathrm{H}$, Takahashi $\mathrm{T}$, and Nomura M, et al (2005) Somatic mutations of the HER2 kinase domain in lung adenocarcinomas Cancer Res 65(5) 1642-1647 https://doi.org/10.1158/0008-5472.CAN-04-4235 PMID: 15753357

66. Yamamoto H, Higasa K, and Sakaguchi M (2013) Novel germline mutation in the transmembrane domain of HER2 in familial lung adenocarcinomas J Natl Cancer Inst 106(1) 1-4

67. Chen $\mathrm{H}, \mathrm{Yu} \mathrm{S}$, and $\mathrm{Ho} \mathrm{B}$, et al (2015) R331W missense mutation of oncogene YAP1 is a germline risk allele for lung adenocarcinoma with medical actionability J Clin Oncol 33(20) 2303-2319 https://doi.org/10.1200/JCO.2014.59.3590 PMID: 26056182

68. Lee T, Tseng Y, and Chang W, et al (2017) YAP1 is essential for tumor growth and is a potential therapeutic target for EGFR-dependent lung adenocarcinomas Oncotarget 8(52) 89539-89551 PMID: 29163769 PMCID: 5685690 
69. Kukita Y, Okami J, and Yoneda-kato N, et al (2016) Homozygous inactivation of CHEK2 is linked to a familial case of multiple primary lung cancer with accompanying cancers in other organs Cold Spring Harb Mol Case Stud 2 1-12 https://doi.org/10.1101/mcs.a001032

70. Madiha K, Xiao-Jie D, and Zhans-Han M, et al (2017) Characterization of germline mutations in familial lung cancer from the Chinese population Gene [http://dx.doi.org/10.1016/j.gene.2017.10.020]

71. Luo W, Tian P, and Wang Y, et al (2018) Characteristics of genomic alterations of lung adenocarcinoma in young never-smokers Int J Cancer 143 1696-1705 https://doi.org/10.1002/ijc.31542 PMID: 29667179 PMCID: 6175072 